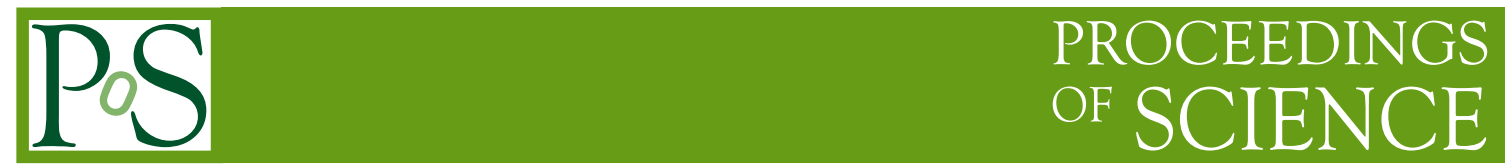

\title{
Theory and Phenomenology of Leptonic CP Violation
}

\section{S. T. Petcov*}

SISSA/INFN, Trieste, Italy, and Kavli IPMU, University of Tokyo (WPI), Tokyo, Japan

E-mail: petcovesissa.it

The current status of our knowledge of the 3-neutrino mixing parameters and of the CP violation in the lepton sector is summarised. The discrete symmetry approach to understanding the observed pattern of neutrino mixing and the related predictions for neutrino mixing angles and leptonic Dirac CP violation are reviewed.

${ }^{*}$ Speaker. 


\section{Introduction: the Three Neutrino Mixing}

It was announced on February 24 this year (2017) that The Pontecorvo Prize for 2016 was awarded to Prof. Yifang Wang (from the Daya Bay Collaboration), Prof. Soo-Bong Kim (from RENO Collaboration ) Prof. K. Nishikawa (from T2K Collaboration) "For their outstanding contributions to the study of the neutrino oscillation phenomenon and to the measurement of the Theta13 mixing angle in the Daya Bay, RENO and T2K experiments." As is well known, the relatively large value of the "reactor" (or "CHOOZ") angle $\theta_{13} \cong 0.15$ measured in the Daya Bay, RENO and Double Chooz experiments, indications for which were obtained first in the T2K experiment 1 , opened up the possibility to search for CP violation effects in neutrino oscillations. Determining the status of CP symmetry in the lepton sector is one of the principal goals of the program of current and future research in neutrino physics. Information on leptonic Dirac CP violation is presently provided by the $\mathrm{T} 2 \mathrm{~K}$ and NOvA neutrino oscillation experiments using as input the reactor neutrino data on $\theta_{13}$ (see, e.g., [1]), and from analyses of the global neutrino oscillation data (see, e.g., $[2,3])$. In the future it is expected to be provided principally by the planned DUNE [4] and T2HK [5] experiments.

Other goals of primal importance of the program of research in neutrino physics, which extends beyond 2030, include [1]:

i) determination of the status of lepton charge conservation and the nature - Dirac or Majorana of massive neutrinos (which is one of the most challenging and pressing problems in present day elementary particle physics);

ii) determination of the spectrum neutrino masses possess, or neutrino mass ordering;

iii) determination of the absolute neutrino mass scale, or $\min \left(m_{j}\right)$.

A successful realisation of this program ${ }^{2}$ is of fundamental importance for making progress in understanding the origin of neutrino masses and mixing and its possible relation to new beyond the Standard Model (BSM) physics.

All compelling neutrino oscillation data is compatible with 3-neutrino mixing in vacuum, which we are going to consider in what follows:

$$
\mathscr{L}_{\mathrm{CC}}=-\frac{g}{\sqrt{2}} \sum_{l=e, \mu, \tau} \overline{l_{L}}(x) \gamma_{\alpha} v_{l L}(x) W^{\alpha \dagger}(x)+\text { h.c. }, v_{l \mathrm{~L}}(x)=\sum_{j=1}^{3} U_{l j} v_{j \mathrm{~L}}(x),
$$

where $v_{l L}(x)$ are the flavour neutrino fields, $v_{j \mathrm{~L}}(x)$ is the left-handed (LH) component of the field of the neutrino $v_{j}$ having a mass $m_{j}$, and $U$ is a unitary matrix - the Pontecorvo, Maki, Nakagawa, Sakata (PMNS) neutrino mixing matrix $[6,7,8], U \equiv U_{\mathrm{PMNS}}$.

In the case of 3 light neutrinos, the $3 \times 3$ unitary neutrino mixing matrix $U$ can be parametrised, as is well known, by 3 angles and, depending on whether the massive neutrinos $v_{j}$ are Dirac or Majorana particles, by one Dirac, or one Dirac and two Majorana, CP violation (CPV) phases [9]:

$$
U=V P, \quad P=\operatorname{diag}\left(1, e^{i \frac{\alpha_{21}}{2}}, e^{i \frac{\alpha_{31}}{2}}\right),
$$

\footnotetext{
${ }^{1}$ For a review of the Daya Bay, RENO, Double Chooz and T2K data on $\theta_{13}$ see, e.g., [1].

${ }^{2}$ See, e.g., [1] for a rather detailed list of current and planned experiments that are foreseen to contribute to the comprehensive long-term program of research in neutrino physics.
} 


\begin{tabular}{lccc}
\hline Parameter & Best fit value & $2 \sigma$ range & $3 \sigma$ range \\
\hline $\sin ^{2} \theta_{12} / 10^{-1}$ & 2.97 & $2.65-3.34$ & $2.50-3.54$ \\
$\sin ^{2} \theta_{13} / 10^{-2}(\mathrm{NO})$ & 2.15 & $1.99-2.31$ & $1.90-2.40$ \\
$\sin ^{2} \theta_{13} / 10^{-2}(\mathrm{IO})$ & 2.16 & $1.98-2.33$ & $1.90-2.42$ \\
$\sin ^{2} \theta_{23} / 10^{-1}(\mathrm{NO})$ & 4.25 & $3.95-4.70$ & $3.81-6.15$ \\
$\sin ^{2} \theta_{23} / 10^{-1}(\mathrm{IO})$ & 5.89 & $3.99-4.83 \oplus 5.33-6.21$ & $3.84-6.36$ \\
$\delta / \pi(\mathrm{NO})$ & 1.38 & $1.00-1.90$ & $0-0.17 \oplus 0.76-2$ \\
$\delta / \pi(\mathrm{IO})$ & 1.31 & $0.92-1.88$ & $0-0.15 \oplus 0.69-2$ \\
\hline$\Delta m_{21}^{2} / 10^{-5} \mathrm{eV}^{2}$ & 7.37 & $7.07-7.73$ & $6.93-7.96$ \\
$\Delta m_{31}^{2} / 10^{-3} \mathrm{eV}^{2}(\mathrm{NO})$ & 2.56 & $2.49-2.64$ & $2.45-2.69$ \\
$\Delta m_{23}^{2} / 10^{-3} \mathrm{eV}^{2}(\mathrm{IO})$ & 2.54 & $2.47-2.62$ & $2.42-2.66$ \\
\hline
\end{tabular}

Table 1: The best fit values, $2 \sigma$ and $3 \sigma$ ranges of the neutrino oscillation parameters obtained in the global analysis of the neutrino oscillation data performed in [2] (The Table is taken from ref. [11]).

where $\alpha_{21,31}$ are the two Majorana CPV phases and in the "standard" parametrisation [1] the matrix $V$ is given by:

$$
V=\left(\begin{array}{ccc}
c_{12} c_{13} & s_{12} c_{13} & s_{13} e^{-i \delta} \\
-s_{12} c_{23}-c_{12} s_{23} s_{13} e^{i \delta} & c_{12} c_{23}-s_{12} s_{23} s_{13} e^{i \delta} & s_{23} c_{13} \\
s_{12} s_{23}-c_{12} c_{23} s_{13} e^{i \delta} & -c_{12} s_{23}-s_{12} c_{23} s_{13} e^{i \delta} & c_{23} c_{13}
\end{array}\right) .
$$

In eq. (1.3), $c_{i j}=\cos \theta_{i j}, s_{i j}=\sin \theta_{i j}$, the angles $\theta_{i j}=[0, \pi / 2)$, and $\delta=[0,2 \pi]$ is the Dirac CPV phase. It follows from the current data that the three massive neutrinos $v_{1,2,3}$ should have masses not exceeding approximately $0.5 \mathrm{eV}, m_{1,2,3} \lesssim 0.5 \mathrm{eV}$. On the basis of the existing neutrino data it is impossible to determine whether the massive neutrinos $v_{j}$ are Dirac or Majorana fermions.

In the case of 3-neutrino mixing, oscillations involving all flavour neutrinos $v_{l}$ (antineutrinos $\left.\bar{v}_{l}\right), v_{l} \leftrightarrow v_{l^{\prime}}\left(\bar{v}_{l} \leftrightarrow \bar{v}_{l^{\prime}}\right), l, l^{\prime}=e, \mu, \tau$, are possible. The 3-neutrino oscillation probabilities $P\left(v_{l} \rightarrow\right.$ $\left.v_{l^{\prime}}\right)$ and $P\left(\bar{v}_{l} \rightarrow \bar{v}_{l^{\prime}}\right)$ are functions of the neutrino energy, $E$, the source-detector distance $L$, of the elements of $U$ and, for relativistic neutrinos used in all neutrino experiments performed so far, of the two independent neutrino mass squared differences $\Delta m_{21}^{2} \neq 0$ and $\Delta m_{31}^{2} \neq 0,\left(\Delta m_{j k}^{2} \equiv m_{j}^{2}-m_{k}^{2}\right)$ present in the case of 3-neutrino mixing (see, e.g., ref. [10]).

The existing data, accumulated over many years of studies of neutrino oscillations, allow us to determine $\Delta m_{21}^{2}, \theta_{12}$, and $\left|\Delta m_{31(32)}^{2}\right|, \theta_{23}$ and $\theta_{13}$, with a relatively high precision [2,3]. Since 2013 there are also persistent hints that the Dirac CPV phase $\delta$ has a value close to $3 \pi / 2$ (see [12]). The best fit values (b.f.v.) and the $2 \sigma$ and $3 \sigma$ allowed ranges of $\Delta m_{21}^{2}, s_{12}^{2},\left|\Delta m_{31(32)}^{2}\right|$, $s_{23}^{2}, s_{13}^{2}$ and $\delta$, found in the latest analysis of global neutrino oscillation data performed in [2] are given in Table 1. Similar results were obtained in ref. [3]. In both analyses [2, 3] the authors find, in particular, that $\sin ^{2} \theta_{23}=0.5$ lies outside the $2 \sigma$ range allowed by the current data, but is within the $3 \sigma$ allowed interval. Both groups also find that the best fit value of the Dirac CPV 
phases $\delta$ is close to $3 \pi / 2$ : in [2], for example, the authors find $\delta=1.38 \pi(1.31 \pi)$ for $\Delta m_{31(32)}^{2}>0$ $\left(\Delta m_{31(32)}^{2}<0\right)$. The absolute $\chi^{2}$ minimum takes place for $\Delta m_{31(32)}^{2}>0$, the local minimum in the case of $\Delta m_{31(32)}^{2}<0$ being approximately by $0.7 \sigma$ higher. According to ref. [2], the CP conserving value $\delta=0$, or $2 \pi$, is disfavored at $2.4 \sigma(3.2 \sigma)$ for $\Delta m_{31(32)}^{2}>0\left(\Delta m_{31(32)}^{2}<0\right)$; the CP conserving value $\delta=\pi$ in the case of $\Delta m_{31(32)}^{2}>0\left(\Delta m_{31(32)}^{2}<0\right)$ is statistically approximately $2.0 \sigma(2.5 \sigma)$ away from the best fit value $\delta \cong 1.38 \pi(1.31 \pi)$. In what concerns the CP violating value $\delta=\pi / 2$, it is strongly disfavored at $3.4 \sigma(3.9 \sigma)$ for $\Delta m_{31(32)}^{2}>0\left(\Delta m_{31(32)}^{2}<0\right)^{3}$. At $3 \sigma, \delta / \pi$ is found to lie in the case of $\Delta m_{31(32)}^{2}>0\left(\Delta m_{31(32)}^{2}<0\right)$ in the following intervals [2]: $(0.00-0.17(0.16)) \oplus(0.76(0.69)-2.00))$. The results on $\delta$ obtained in [3] differ somewhat from, but are compatible at $1 \sigma$ C.L. with, those found in [2].

It follows also from the results quoted in Table 1 that $\Delta m_{21}^{2} /\left|\Delta m_{31(32)}^{2}\right| \cong 0.03$. We have $\left|\Delta m_{31}^{2}\right|=\left|\Delta m_{32}^{2}-\Delta m_{21}^{2}\right| \cong\left|\Delta m_{32}^{2}\right|$. The angle $\theta_{12}$ is definitely smaller than $\pi / 4$ : the value of $\theta_{12}=\pi / 4$, i.e., maximal solar neutrino mixing, is ruled out at high confidence level by the data: $\cos 2 \theta_{12} \geq 0.29$ at $99.73 \%$ C.L. The quoted results imply also that the value of $\theta_{23}$ can deviate by approximately \pm 0.1 from $\pi / 4, \theta_{12} \cong \pi / 5.4$ and that $\theta_{13} \cong \pi / 20$. Thus, the pattern of neutrino mixing differs drastically from the pattern of quark mixing.

Apart from the hint that the Dirac phase $\delta \sim 3 \pi / 2$, no other experimental information on the Dirac and Majorana CPV phases in the neutrino mixing matrix is available at present. Thus, the status of CP symmetry in the lepton sector is essentially unknown. With $\theta_{13} \cong 0.15 \neq 0$, the Dirac phase $\delta$ can generate $\mathrm{CP}$ violating effects in neutrino oscillations [9, 13], i.e, a difference between the probabilities of the $v_{l} \rightarrow v_{l^{\prime}}$ and $\bar{v}_{l} \rightarrow \bar{v}_{l^{\prime}}$ oscillations, $l \neq l^{\prime}=e, \mu, \tau$. The magnitude of CP violation in $v_{l} \rightarrow v_{l^{\prime}}$ and $\bar{v}_{l} \rightarrow \bar{v}_{l^{\prime}}$ oscillations, $l \neq l^{\prime}=e, \mu, \tau$, is determined by [14] the rephasing invariant $J_{C P}$, associated with the Dirac CPV phase in $U$ :

$$
J_{\mathrm{CP}}=\operatorname{Im}\left(U_{\mu 3} U_{e 3}^{*} U_{e 2} U_{\mu 2}^{*}\right) .
$$

It is analogous to the rephasing invariant associated with the Dirac CPV phase in the CKM quark mixing matrix [15]. In the standard parametrisation of the neutrino mixing matrix (1.3), $J_{\mathrm{CP}}$ has the form:

$$
J_{C P} \equiv \operatorname{Im}\left(U_{\mu 3} U_{e 3}^{*} U_{e 2} U_{\mu 2}^{*}\right)=\frac{1}{8} \cos \theta_{13} \sin 2 \theta_{12} \sin 2 \theta_{23} \sin 2 \theta_{13} \sin \delta .
$$

Thus, given the fact that $\sin 2 \theta_{12}, \sin 2 \theta_{23}$ and $\sin 2 \theta_{13}$ have been determined experimentally with a relatively high precision, the size of $\mathrm{CP}$ violation effects in neutrino oscillations depends essentially only on the magnitude of the currently not well determined value of the Dirac phase $\delta$. The current data implies $0.026(0.027)|\sin \delta| \lesssim\left|J_{C P}\right| \lesssim 0.035|\sin \delta|$, where we have used the $3 \sigma$ ranges of $\sin ^{2} \theta_{12}, \sin ^{2} \theta_{23}$ and $\sin ^{2} \theta_{13}$ given in Table 1. For the current best fit values of $\sin ^{2} \theta_{12}, \sin ^{2} \theta_{23}$, $\sin ^{2} \theta_{13}$ and $\delta$ we find in the case of $\Delta m_{31(2)}^{2}>0\left(\Delta m_{31(2)}^{2}<0\right): J_{C P} \cong 0.032 \sin \delta \cong-0.030$ $\left(J_{C P} \cong 0.032 \sin \delta \cong-0.027\right)$. Thus, if the indication that $\delta$ has a value close to $3 \pi / 2$ is confirmed by future more precise data, i) the $J_{C P}$ factor in the lepton sector would be approximately by 3 orders of magnitude larger in absolute value than the corresponding $J_{C P}$ factor in the quark sector, and ii) the $\mathrm{CP}$ violation effects in neutrino oscillations would be relatively large and observable.

\footnotetext{
${ }^{3}$ The quoted confidence levels for $\delta=0, \pi$ and $\pi / 2$ are all with respect to the absolute $\chi^{2}$ minimum.
} 
If the neutrinos with definite masses $v_{i}, i=1,2,3$, are Majorana particles, the 3-neutrino mixing matrix contains two additional Majorana CPV phases [9]. However, the flavour neutrino oscillation probabilities $P\left(v_{l} \rightarrow v_{l^{\prime}}\right)$ and $P\left(\bar{v}_{l} \rightarrow \bar{v}_{l^{\prime}}\right), l, l^{\prime}=e, \mu, \tau$, do not depend on the Majorana phases $[9,16]$. The Majorana phases can play important role, e.g, in $|\Delta L|=2$ processes like neutrinoless double beta $\left((\beta \beta)_{0 v^{-}}\right)$decay $(A, Z) \rightarrow(A, Z+2)+e^{-}+e^{-}, L$ being the total lepton charge, in which the Majorana nature of massive neutrinos $v_{i}$ manifests itself (see, e.g, refs. [10, 17]).

Our interest in the CPV phases present in the neutrino mixing matrix is stimulated also by the intriguing possibility that the Dirac phase and/or the Majorana phases in $U_{\mathrm{PMNS}}$ can provide the CP violation necessary for the generation of the observed baryon asymmetry of the Universe (BAU) [18] (for specific models in which this possibility is realised see, e.g., [19]).

Understanding the origin of the patterns of neutrino mixing and of neutrino mass squared differences, revealed by the data obtained in the neutrino oscillation experiments is one of the most challenging problems in neutrino physics. It is part of the more general fundamental problem in particle physics of understanding the origins of flavour, i.e., of the patterns of quark, charged lepton and neutrino masses, and of the quark and lepton mixing.

In this article we will review aspects of the SYMMETRY approach to understanding the form of neutrino mixing, which is based on non-Abelian discrete flavour symmetries and is widely explored at present (see, e.g., [20, 21, 22] and references therein). One of the most striking features of this approach is that it leads to specific correlations between the values of at least some of the mixing angles of the neutrino mixing matrix $U_{\mathrm{PMNS}}$ and, either to specific fixed values of CPV phases present in $U_{\text {PMNS }}$, which are "trivial" (e.g., $\delta=0$ or $\pi, \alpha_{21}=\alpha_{31}=0$ ), ( see, e.g., [22]), or to a correlation between the values of the neutrino mixing angles and of the cosine of the Dirac CPV phase $\delta$ of $U_{\text {PMNS }}[23,24,25,26]^{4}$, i.e., to a "sum rule" for $\cos \delta$. As a consequence of this correlation one obtains predictions for the value of $\delta$ and, correspondingly, for the $J_{\mathrm{CP}}$ factor and for the $\mathrm{CP}$ violating effects in neutrino oscillations. These predictions depend, in particular, on the the underlying discrete symmetry used to derive the observed pattern of neutrino mixing and on the type of breaking of the symmetry, necessary to reproduce the measured values of the neutrino mixing angles. We will review also the predictions for $\delta$ and the $J_{\mathrm{CP}}$ factor in the cases of widely discussed underlying symmetry patterns of the PMNS matrix and the prospects of testing these predictions in future planned neutrino oscillation experiments.

\section{Discrete Symmetry Approach to Neutrino Mixing (The Quest for Nature's Message)}

We believe, and we are not alone in holding this view, that with the observed pattern of neutrino mixing Nature is "sending" us a Message. The Message is encoded in the values of the neutrino mixing angles, leptonic CPV phases in the PMNS matrix and neutrino masses. We do not know at present what is the content of Nature's Message. However, on the basis of the current ideas about the possible origins of the observed pattern of neutrino mixing, the Nature's Message can have two

\footnotetext{
${ }^{4}$ In the case of Majorana massive neutrinos one can obtain (under specific conditions) also correlations between the values of the two Majorana CPV phases present in $U_{\mathrm{PMNS}}$ and of the three neutrino mixing angles and of the Dirac CPV phase [23].
} 
completely different contents, each of which can be characterised by one word: ANARCHY or SYMMETRY. In the ANARCHY approach [27] to understanding the pattern of neutrino mixing it is assumed that Nature "threw dice" when Nature was "choosing" the values of the neutrino masses, mixing angles and leptonic CPV phases. The main prediction of the ANARCHY explanation of the pattern of neutrino mixing is the absence of whatever correlations between the values of the neutrino mixing angles, between the values of the neutrino mixing angles and the CPV phases and between the values of the neutrino masses, all of them being random quantities. In contrast, one of the most characteristic prediction of the SYMMETRY approach to neutrino mixing is the existence of correlations between the values of at least some of the observables (angles, CPV phases) of the the neutrino mixing matrix.

Within the SYMMETRY approach, the observed pattern of neutrino mixing can be naturally understood on the basis of specific class of symmetries - the class of non-Abelian discrete (finite) flavour symmetries (see, e.g., [20, 21, 22]). Thus, the specific form of the neutrino mixing can have its origin in the existence of new fundamental symmetry in the lepton sector. The most distinctive feature of the approach to neutrino mixing based on non-Abelian discrete flavour symmetries is the predictions i) of the values of some of the neutrino mixing angles and leptonic CPV phases, and/or ii) of existence of correlations between the values of at least some the neutrino mixing angles and/or between the values of the neutrino mixing angles and the Dirac CPV phase in the PMNS matrix, etc. (see, e.g., $[22,23,24,25])^{5}$. Most importantly, these predictions and predicted correlations, and thus the discrete symmetry approach itself, can be tested experimentally (see, e.g., [23] and $[11,24,33,34,35])$.

\subsection{Symmetry Forms of Neutrino Mixing}

The observed pattern of neutrino mixing in the reference 3-neutrino mixing scheme we are going to consider in what follows is characterised, as we have seen, by two large mixing angles $\theta_{12}$ and $\theta_{23}$, and one small mixing angle $\theta_{13}: \theta_{12} \cong 33^{\circ}, \theta_{23} \cong 45^{\circ} \pm 6^{\circ}$ and $\theta_{13} \cong 8.4^{\circ}$. These values can naturally be explained by extending the Standard Theory (ST) with a flavour symmetry corresponding to a non-Abelian discrete (finite) group $G_{f}$. This symmetry is supposed to exist at some high-energy scale and to be broken at lower energies to residual symmetries of the charged lepton and neutrino sectors, described respectively by subgroups $G_{e}$ and $G_{v}$ of $G_{f}$. Flavour symmetry groups $G_{f}$ that have been used in this approach to neutrino mixing and lepton flavour include $S_{4}$, $A_{4}, T^{\prime}, A_{5}, D_{n}$ (with $n=10,12$ ), $\Delta(27)$, the series $\Delta\left(6 n^{2}\right)$, to name several ${ }^{6}$ (see, e.g., ref. [21] for definitions of these groups and discussion of their properties ${ }^{7}$ ). The numbers of elements, of generators and of irreducible representations of the groups $S_{4}, A_{4}, T^{\prime}, A_{5}, D_{10}$ and $D_{12}$ are given in Table 2. The choice of the non-Abelian discrete groups $S_{4}, A_{4}, T^{\prime}, A_{5}$, etc. is related, in par-

\footnotetext{
${ }^{5}$ Combining the discrete symmetry approach with the idea of generalised CP invariance $[28,29]$ - a generalistaion of the standard CP invariance requirement - allows to obtain predictions also for the Majorana CPV phases in the PMNS matrix in the case of massive Majorana neutrinos (see, e.g., [11, 30, 31, 32] and references quoted therein).

${ }^{6}$ Some of the groups $T^{\prime}, A_{5}$, etc. can be and have been used also for a unified description of the quark and lepton flavours, see, e.g., refs. [36] and references quoted therein.

${ }^{7} S_{4}$ is the group of permutations of 4 objects and the symmetry group of the cube. $A_{4}$ is the group of even permutations of 4 objects and the symmetry group of the regular tetrahedron. $T^{\prime}$ is the double covering group of $A_{4}$. $A_{5}$ is the icosahedron symmetry group of even permutations of five objects, etc. All these groups are subgroups of $S U(3)$ and this will be assumed to hold for $G_{f}$ considered by us.
} 


\begin{tabular}{|c|c|c|c|}
\hline Group & Number of elements & Generators & Irreducible representations \\
\hline$S_{4}$ & 24 & $S, T, U$ & $\mathbf{1}, \mathbf{1}^{\prime}, \mathbf{2}, \mathbf{3}, \mathbf{3}^{\prime}$ \\
$A_{4}$ & 12 & $S, T$ & $\mathbf{1}, \mathbf{1}^{\prime}, \mathbf{1}^{\prime \prime}, \mathbf{3}$ \\
$T^{\prime}$ & 24 & $S, T, R$ & $\mathbf{1}^{\prime}, \mathbf{1}^{\prime \prime}, \mathbf{2}, \mathbf{2}^{\prime}, \mathbf{2}^{\prime \prime}, \mathbf{3}$ \\
$A_{5}$ & 60 & $S, T$ & $\mathbf{1}, \mathbf{3}, \mathbf{3}^{\prime}, \mathbf{4}, \mathbf{5}$ \\
$D_{10}$ & 20 & $A, B$ & $\mathbf{1}_{1}, \mathbf{1}_{2}, \mathbf{1}_{3}, \mathbf{1}_{4}, \mathbf{2}_{1}, \mathbf{2}_{2}, \mathbf{2}_{3}, \mathbf{2}_{4}$ \\
$D_{12}$ & 24 & $A, B$ & $\mathbf{1}_{1}, \mathbf{1}_{2}, \mathbf{1}_{3}, \mathbf{1}_{4}, \mathbf{2}_{1}, \mathbf{2}_{2}, \mathbf{2}_{3}, \mathbf{2}_{4}, \mathbf{2}_{5}$ \\
\hline
\end{tabular}

Table 2: Number of elements, generators and irreducible representations of some discrete groups.

ticular, to the fact that they describe symmetries with respect to rotations on fixed large mixing angles and, correspondingly, lead to values of the neutrino mixing angles $\theta_{12}$ and $\theta_{23}$, which can differ from the measured values at most by sub-leading perturbative corrections, with $\theta_{13}$ typically (but not universally) predicted to be zero. For instance, the groups $A_{4}, S_{4}$ and $T^{\prime}$ are commonly utilised to generate tri-bimaximal (TBM) mixing [37]; the group $S_{4}$ can also be used to generate bimaximal (BM) mixing ${ }^{8}$ [39]; $A_{5}$ can be utilised to generate golden ratio type A (GRA) [40] mixing; and the groups $D_{10}$ and $D_{12}$ can lead to golden ratio type B (GRB) [41] and hexagonal (HG) [42] mixing. For all these symmetry forms the neutrino mixing matrix $U_{v}^{\circ}$ has the form: $U_{v}^{\circ}=R_{23}\left(\theta_{23}^{v}\right) R_{13}\left(\theta_{13}^{v}\right) R_{12}\left(\theta_{12}^{v}\right)$ with $\theta_{23}^{v}=-\pi / 4$ and $\theta_{13}^{v}=0$ :

$$
U_{v}^{\circ}=R_{23}\left(\theta_{23}^{v}=-\pi / 4\right) R_{12}\left(\theta_{12}^{v}\right)=\left(\begin{array}{ccc}
\cos \theta_{12}^{v} & \sin \theta_{12}^{v} & 0 \\
-\frac{\sin \theta_{12}^{v}}{\sqrt{2}} & \frac{\cos \theta_{12}^{v}}{\sqrt{2}} & -\frac{1}{\sqrt{2}} \\
-\frac{\sin \theta_{12}^{v}}{\sqrt{2}} & \frac{\cos \theta_{12}^{v}}{\sqrt{2}} & \frac{1}{\sqrt{2}}
\end{array}\right) .
$$

The value of the angle $\theta_{12}^{v}$, and thus of $\sin ^{2} \theta_{12}^{v}$, depends on the symmetry form of $U_{v}^{\circ}$. For the TBM, BM, GRA, GRB and HG forms we have: i) $\sin ^{2} \theta_{12}^{v}=1 / 3$ (TBM), ii) $\sin ^{2} \theta_{12}^{v}=1 / 2$ (BM), iii) $\sin ^{2} \theta_{12}^{v}=(2+r)^{-1} \cong 0.276$ (GRA), $r$ being the golden ratio, $r=(1+\sqrt{5}) / 2$, iv) $\sin ^{2} \theta_{12}^{v}=$ $(3-r) / 4 \cong 0.345$ (GRB), and $\mathrm{v}) \sin ^{2} \theta_{12}^{v}=1 / 4(\mathrm{HG})$.

In the approach under discussion it is standardly assumed that the LH neutrino fields, $v_{\tilde{l} L}(x)$, and the LH components of the charged lepton fields (in the basis in which charged lepton mass term is not diagonal) $\tilde{l}_{L}(x)$, which form an $S U(2)_{L}$ doublet in the Standard Theory, are assigned to the same r-dimensional irreducible unitary representation $\rho_{r}\left(g_{f}\right)$ of the Group $G_{f}, g_{f}$ being an element of $G_{f}$. In the cases of $G_{f}=A_{4}, S_{4}, T^{\prime}$ and $A_{5}$, which possess 3-dimensional irreducible representations, $\rho\left(g_{f}\right)$ is standardly taken to be a 3-dimensional irreducible unitary representation $\mathbf{3}, \rho_{r}\left(g_{f}\right)=\rho_{3}\left(g_{f}\right)$. This is equivalent to the assumption of unification of the three lepton families at some high energy scale. We are going to consider this choice in what follows.

\footnotetext{
${ }^{8}$ Bimaximal mixing can also be a consequence of the conservation of the lepton charge $L^{\prime}=L_{e}-L_{\mu}-L_{\tau}$ (LC) [38], supplemented by $\mu-\tau$ symmetry.
} 
At low energies the flavour symmetry $G_{f}$ has necessarily to be broken so that the three lepton flavours can be distinguished and the electron, muon and tauon as well as the three neutrinos with definite mass $v_{1}, v_{2}$ and $v_{3}$, can get different masses. Thus, $G_{f}$ is broken to different residual symmetries $G_{e}$ and $G_{v}, G_{e} \neq G_{v}$, of the charged lepton and neutrino mass terms, respectively 9. Possible discrete symmetries $G_{e}$ of the charged lepton mass term (leaving $M_{e} M_{e}^{\dagger}$ invariant, $M_{e}$ being the charged lepton mass matrix in left-right (L-R) convention) are: i) $G_{e}=Z_{n}$, with integer $n \geq 2$, or ii) $Z_{m} \times Z_{k}$, with integer $m, k \geq 2$. The maximal symmetry $G_{v}$ of the Majorana mass term of the LH flavour neutrino fields $v_{\tilde{l} L}(x)$ (leaving $M_{v}$ and $M_{v}^{\dagger} M_{v}$ invariant, $M_{v}$ being the mass matrix in R-L convention) is the $Z_{2} \times Z_{2}$ (sometimes referred to as the Klein four group) symmetry. $G_{v}$ can obviously be just $Z_{2}$. The subgroup $G_{e}$, in particular, can be trivial.

For fixed $G_{f}$ and irreducible representation $\rho_{r}\left(g_{f}\right)$, non-trivial residual symmetries constrain the forms of the $3 \times 3$ unitary matrices $U_{e}$ and $U_{v}$, which diagonalise the charged lepton and neutrino mass matrices, and the product of which represents the PMNS matrix:

$$
U_{\mathrm{PMNS}}=U_{e}^{\dagger} U_{v}
$$

Thus, the residual symmetries constrain also the form of $U_{\mathrm{PMNS}}$.

The TBM form of $U_{v}^{\circ}$ (see eq. (2.1)) can originate from $G_{f}=S_{4}$ symmetry with residual symmetry $G_{v}=Z_{2} \times Z_{2}$; it can be obtained also from a $G_{f}=A_{4}$ symmetry with $G_{v}=Z_{2}$ and imposing additional "accidental" $\mu-\tau$ (i.e., $Z_{2}$ ) symmetry of the neutrino Majorana mass matrix $M_{v}$ (see, e.g., [22] and references quoted therein). The group $G_{f}=S_{4}$ can also be used to generate the BM from of $U_{v}^{\circ}$ (e.g., by choosing $G_{v}=Z_{2}$ combined with an accidental $\mu-\tau$ symmetry) $[43,25]$. In all these cases $U_{v}=U_{v}^{\circ} P^{\circ}, P^{\circ}=P^{\circ}\left(\xi_{21}, \xi_{31}\right)$ being a diagonal matrix containing two phases $\xi_{21}$ and $\xi_{31}$ which contribute to the Majorana phases $\alpha_{21}$ and $\alpha_{31}$.

In the symmetry approach to neutrino mixing typically the matrix $U_{v}$ has an underlying symmetry form, for example, TBM, BM, GRA, GRB, HG. For all these five forms $U_{v}=U_{v}^{\circ} P^{\circ}, \theta_{13}^{v}=0$ and needs to be corrected; if the measured $\theta_{23}$ is established to differ significantly from $\pi / 4$, $\left|\theta_{23}^{v}\right|=\pi / 4$ should be corrected. The sub-leading perturbative corrections, needed to bring the "symmetry" values of the three neutrino mixing angles in $U_{v}^{\circ}$ to the measured values of $\theta_{12}, \theta_{23}$ and $\theta_{13}$ in $U_{\mathrm{PMNS}}$ can most naturally be provided by the unitary matrix $U_{e}$ (see, e.g., [26]). In certain classes of models, however, $U_{e}$ coincides with the unit $3 \times 3$ matrix and the requisite corrections are incorporated in a factor contained in the matrix $U_{v}$ (see, e.g., [22]).

As we have indicated, one of the main characteristics of the discussed approach to neutrino mixing based on discrete flavour symmetries is that it leads to certain specific predictions for the values of, and/or correlations between, the low-energy neutrino mixing parameters, which can be tested experimentally. These predictions depend on the chosen $G_{f}, \rho\left(g_{f}\right), G_{e}$ and $G_{v}$. We give a few examples [22, 23, 24, 25, 29, 30, 31, 32, 34, 43].

I. In a large class of models one gets $\sin ^{2} \theta_{23}=0.5$.

II. In different class of models one finds that the values of $\sin ^{2} \theta_{23}$ and $\sin ^{2} \theta_{13}$ are correlated: $\sin ^{2} \theta_{23}=0.5\left(1 \mp \sin ^{2} \theta_{13}+O\left(\sin ^{4} \theta_{13}\right)\right)$.

III. In certain models $\sin ^{2} \theta_{23}$ is predicted to have specific values which differ significantly from

\footnotetext{
${ }^{9}$ Given a discrete (finite) $G_{f}$, there are more than one (but still a finite number of) possible residual symmetries $G_{e}$ and $G_{V}$, see, e.g., [21, 22].
} 
those in cases I and II [24]: $\sin ^{2} \theta_{23}=0.455$; or 0.463 ; or 0.537 ; or 0.545 , the uncertainties in these predictions being insignificant.

IV. Certain class of models predict a correlation between the values of $\sin ^{2} \theta_{12}$ and $\sin ^{2} \theta_{13}: \sin ^{2} \theta_{12}=$ $1 /\left(3 \cos ^{2} \theta_{13}\right)=\left(1+\sin ^{2} \theta_{13}+O\left(\sin ^{4} \theta_{13}\right)\right) / 3 \cong 0.340$, where we have used the b.f.v. of $\sin ^{2} \theta_{13}$.

V. In another class of models one still finds a correlation between the values of $\sin ^{2} \theta_{12}$ and $\sin ^{2} \theta_{13}$, which, however, differs from that in Case IV: $\sin ^{2} \theta_{12}=\left(1-3 \sin ^{2} \theta_{13}\right) /\left(3 \cos ^{2} \theta_{13}\right)=$ $\left(1-2 \sin ^{2} \theta_{13}+O\left(\sin ^{4} \theta_{13}\right)\right) / 3 \cong 0.319$, where we have used again the b.f.v. of $\sin ^{2} \theta_{13}$.

VI. In large classes of models in which the elements of the PMNS matrix are predicted to be functions of just one real continuous free parameter ("one-parameter models"), the Dirac and the Majorana CPV phases have "trivial" CP conserving values 0 or $\pi$. In certain one-parameter schemes, however, the Dirac phases $\delta=\pi / 2$ or $3 \pi / 2$.

VII. In models in which the elements of the PMNS matrix are functions of two (angle, or one angle and one phase) or three (two angle and one phase) parameters, the Dirac phase $\delta$ satisfies a sum rule by which $\cos \delta$ is expressed in terms of the three neutrino mixing angles $\theta_{12}, \theta_{23}, \theta_{13}$ and one (or more) fixed (known) parameters $\theta^{v}$ which depend of the discrete symmetry $G_{f}$ employed and on the residual symmetries $G_{e}$ and $G_{v}[23,24,25,26]$. In this cases the $J_{C P}$ factor which determines the magnitude of $\mathrm{CP}$ violation effects in neutrino oscillations, is also completely determined by the values of the three neutrino mixing angles and the symmetry parameter(s) $\theta_{v}$.

The predictions listed above, and therefore the respective models can be and will be tested in the currently running (T2K, NOvA) and future planned (JUNO, T2HK, DUNE) experiments.

\subsection{Predictions for the Dirac CPV Phase}

We will consider next for concreteness the approach followed in $[23,33,26,24]$ in which the requisite corrections to the underlying symmetry form of the neutrino mixing matrix are provided by the matrix $U_{e}$ corresponding to $G_{f}$ i) either broken to $G_{e}=Z_{2}$, or ii) completely broken, by the charged lepton mass term. In this case the PMNS matrix has the following general form [44]:

$$
U=U_{e}^{\dagger} U_{v}=\left(\tilde{U}_{e}\right)^{\dagger} \Psi U_{v}^{\circ} P^{\circ}
$$

Here $\tilde{U}_{e}$ is a $3 \times 3$ unitary matrix and $\Psi$ is a diagonal phase matrix. The matrix $\tilde{U}_{e}$ was chosen in $[23,33,26]$ to have the following two forms:

$$
\mathbf{A}: \tilde{U}_{e}=R_{23}^{-1}\left(\theta_{23}^{e}\right) R_{12}^{-1}\left(\theta_{12}^{e}\right) ; \mathbf{B}: \tilde{U}_{e}=R_{12}^{-1}\left(\theta_{12}^{e}\right) .
$$

where $\theta_{12}^{e}$ and $\theta_{23}^{e}$ are free real angle parameters. These two forms appear in a large class of theoretical models of flavour and studies, in which the generation of charged lepton masses is an integral part (see, e.g., [30, 45]). The phase matrix $\Psi$ in cases $\mathbf{A}$ and $\mathbf{B}$ is given by [23, 26]:

$$
\mathbf{A}: \Psi=\operatorname{diag}\left(1, e^{-i \psi}, e^{-i \omega}\right) ; \mathbf{B}: \Psi=\operatorname{diag}\left(1, e^{-i \psi}, 1\right) .
$$

The phases $\omega$ and/or $\psi$ serve as a source for the Dirac CPV phase $\delta$ of the PMNS matrix and contribute to the Majorana CPV phases of the PMNS matrix $\alpha_{21}$ and $\alpha_{31}$ [23]. The diagonal phase matrix $P^{\circ}$ in eq. (2.3) contains two phases, $\xi_{21}$ and $\xi_{31}$, which also contribute to the Majorana phases $\alpha_{21}$ and $\alpha_{31}$, respectively.

Consider first the case of the five underlying symmetry forms of $U_{v}^{\circ}$ - TBM, BM, GRA, GRB and HG - corrected by the matrix $U_{e}$, with the PMNS matrix given in eq. (2.3) and the matrices $\tilde{U}_{e}$ 
and $\Psi$ as given in eqs. (2.4) and (2.5). In the considered setting the Dirac phase $\delta$ of the PMNS matrix satisfies the following sum rule [23]:

$$
\cos \delta=\frac{\tan \theta_{23}}{\sin 2 \theta_{12} \sin \theta_{13}}\left[\cos 2 \theta_{12}^{v}+\left(\sin ^{2} \theta_{12}-\cos ^{2} \theta_{12}^{v}\right)\left(1-\cot ^{2} \theta_{23} \sin ^{2} \theta_{13}\right)\right] .
$$

Within the approach employed this sum rule is exact and is valid for any value of the angle $\theta_{23}^{v}$ [24] (and not only for $\theta_{23}^{v}=-\pi / 4$ of the five discussed symmetry forms of $U_{v}^{\circ}$ ). As we see, via the sum rule $\cos \delta$ is expressed in terms of the three neutrino mixing angles $\theta_{12}, \theta_{23}, \theta_{13}$ and one fixed (known) parameter $\theta^{v}$ which depends on the underlying symmetry form (TBM, BM, GRA, GRB, HG) of the PMNS matrix. The difference between the cases $\mathbf{A}$ and $\mathbf{B}$ of forms of $\tilde{U}_{e}$ in eq. (2.4) is, in particular, in the correlation between the values of $\sin ^{2} \theta_{23}$ and $\sin ^{2} \theta_{13}$ they lead to. In case $\mathbf{A}$ of $\tilde{U}_{e}$ the values of $\sin ^{2} \theta_{23}$ and $\sin ^{2} \theta_{13}$ are not correlated and $\sin ^{2} \theta_{23}$ can differ significantly from 0.5 [23]. For the form $\mathbf{B}$ of $\tilde{U}_{e}$ we have [23]:

$$
\sin ^{2} \theta_{23}=\frac{1}{2} \frac{1-2 \sin ^{2} \theta_{13}}{1-\sin ^{2} \theta_{13}} \cong \frac{1}{2}\left(1-\sin ^{2} \theta_{13}\right) .
$$

Thus, in contrast to the case $\mathbf{A}$, in case $\mathbf{B}$ the value of $\sin ^{2} \theta_{23}$ is correlated with the value of $\sin ^{2} \theta_{13}$ and as a consequence $\sin ^{2} \theta_{23}$ can deviate from 0.5 insignificantly - only by $0.5 \sin ^{2} \theta_{13}$.

Given the values of $\sin \theta_{23}, \sin \theta_{23}, \sin \theta_{13}$ and $\theta_{12}^{v}, \cos \delta$ is determined uniquely by the sum rule (2.6). This allows us to determine also $|\sin \delta|$ uniquely, but not $\operatorname{sgn}(\sin \delta)$, which leads to a two-fold ambiguity in the predicted value of $\delta$.

The fact that the value of the Dirac CPV phase $\delta$ is determined (up to an ambiguity of the sign of $\sin \delta$ ) by the values of the three mixing angles $\theta_{12}, \theta_{23}$ and $\theta_{13}$ of the PMNS matrix and the value of $\theta_{12}^{v}$ of the matrix $U_{v}^{\circ}$, eq. (2.1), is the most striking prediction of the models considered. This result implies that in the schemes under discussion, the rephasing invariant $J_{\mathrm{CP}}$ associated with the Dirac phase $\delta$, eq. (1.5), is also a function of the three angles $\theta_{12}, \theta_{23}$ and $\theta_{13}$ of the PMNS matrix and of $\theta_{12}^{v}$ :

$$
J_{\mathrm{CP}}=J_{\mathrm{CP}}\left(\theta_{12}, \theta_{23}, \theta_{13}, \delta\left(\theta_{12}, \theta_{23}, \theta_{13}, \theta_{12}^{v}\right)\right)=J_{\mathrm{CP}}\left(\theta_{12}, \theta_{23}, \theta_{13}, \theta_{12}^{v}\right) .
$$

This allows to obtain predictions for the possible values of $J_{\mathrm{CP}}$ for the different symmetry forms of $U_{v}^{\circ}$ (specified by the value of $\theta_{12}^{v}$ ) using the current data on $\theta_{12}, \theta_{23}$ and $\theta_{13}$.

In [23], by using the sum rule in eq. (2.6), predictions for $\cos \delta, \delta$ and the $J_{\mathrm{CP}}$ factor were obtained in the TBM, BM, GRA, GRB and HG cases for the b.f.v. of $\sin ^{2} \theta_{12}, \sin ^{2} \theta_{23}$ and $\sin ^{2} \theta_{13}$. It was found that the predictions of $\cos \delta$ vary significantly with the symmetry form of $U_{v}^{\circ}$. For the b.f.v. of $\sin ^{2} \theta_{12}=0.308, \sin ^{2} \theta_{13}=0.0234$ and $\sin ^{2} \theta_{23}=0.437$ found for NO spectrum in [12], for instance, one gets [23] $\cos \delta=(-0.0906),(-1.16), 0.275,(-0.169)$ and 0.445 , for the TBM, BM (LC), GRA, GRB and HG forms, respectively. For the TBM, GRA, GRB and HG forms these values correspond to $\delta= \pm 95.2^{\circ}, \pm 74.0^{\circ}, \pm 99.7^{\circ}, \pm 63.6^{\circ}$. For the b.f.v. given in Table 1 and obtained in the recent global analysis [2] one finds in the cases of the TBM, BM (LC), GRA, GRB and HG forms the values given in Table 3. Due to the different NO and IO b.f.v. of $\sin ^{2} \theta_{23}$, the predicted values of $\cos \delta$ and $\delta$ for IO spectrum differ (in certain cases significantly) from those for the NO spectrum. 
Table 3: Predicted values of $\cos \delta$ and $\delta$ for the five symmetry forms, TBM, BM, GRA, GRB and HG, and $\tilde{U}_{e}$ given by the form $\mathbf{A}$ in eq. (2.4), obtained using eq. (2.6) and the best fit values of $\sin ^{2} \theta_{12}, \sin ^{2} \theta_{23}$ and $\sin ^{2} \theta_{13}$ for NO and IO neutrino mass spectra from ref. [2].

\begin{tabular}{lcccc}
\hline Scheme & $\cos \delta(\mathrm{NO})$ & $\delta(\mathrm{NO})$ & $\cos \delta(\mathrm{IO})$ & $\delta(\mathrm{IO})$ \\
\hline TBM & -0.16 & $\pm 99^{\circ}$ & -0.27 & $\pm 106^{\circ}$ \\
BM (LC) & -1.26 & $\delta$-unphysical & -1.78 & $\delta$-unphysical \\
GRA & 0.21 & $\pm 78^{\circ}$ & 0.24 & $\pm 76^{\circ}$ \\
GRB & -0.24 & $\pm 105^{\circ}$ & -0.38 & $\pm 112^{\circ}$ \\
HG & 0.39 & $\pm 67^{\circ}$ & 0.48 & $\pm 62^{\circ}$ \\
\hline
\end{tabular}

Two comments are in order. First, according to the results found in [2] and quoted in Table 1, the predicted values of $\delta$ lying in the first quadrant are strongly disfavored (if not ruled out) by the current data. Second, the unphysical value of $\cos \delta$ in the BM (LC) case is a reflection of the fact that the scheme under discussion with BM (LC) form of the matrix $U_{v}^{\circ}$ does not provide a good description of the current data on $\theta_{12}, \theta_{23}$ and $\theta_{13}$ [26]. Physical values of $\cos \delta$ can be obtained in the case of the NO spectrum, e.g., for the b.f.v. of $\sin ^{2} \theta_{13}$ if the value of $\sin ^{2} \theta_{12}\left(\sin ^{2} \theta_{23}\right)$ is larger (smaller) than the current best fit value ${ }^{10}[23,33]$. However, with the current b.f.v. of $\sin ^{2} \theta_{23}$ in the case of IO spectrum, the BM (LC) form is strongly disfavored.

The results quoted above imply [23] that a measurement of $\cos \delta$ can allow to distinguish between at least some of the different symmetry forms of $U_{v}^{\circ}$, provided $\theta_{12}, \theta_{13}$ and $\theta_{23}$ are known, and $\cos \delta$ is measured, with sufficiently high precision ${ }^{11}$. Even determining the sign of $\cos \delta$ will be sufficient to eliminate some of the possible symmetry forms of $U_{v}^{\circ}$.

These conclusions were confirmed by the statistical analyses performed in ref. [33] where predictions of the sum rule (2.6) for i) $\delta, \cos \delta$ and the rephasing invariant $J_{\mathrm{CP}}$ using the "data" (best fit values and $\chi^{2}$-distributions) on $\sin ^{2} \theta_{12}, \sin ^{2} \theta_{13}, \sin ^{2} \theta_{23}$ and $\delta$ from [12], and ii) for $\cos \delta$, using prospective uncertainties on $\sin ^{2} \theta_{12}, \sin ^{2} \theta_{13}$ and $\sin ^{2} \theta_{23}$, were derived for the TBM, BM (LC), GRA, GRB and HG symmetry forms of the matrix $U_{v}^{\circ}$. Both analyses were performed for the case of NO neutrino mass spectrum. The results for the IO spectrum are similar. The aim of the first analysis, the results of which for $J_{\mathrm{CP}}$ are shown in Fig. 1, was to derive the allowed ranges for $\delta$ and $J_{\mathrm{CP}}$, predicted on the basis of the current data on the neutrino mixing parameters for each of the symmetry forms of $U_{v}^{\circ}$ considered (see [33] for details of the analysis). We have found [33], in particular, that the CP-conserving value of $J_{\mathrm{CP}}=0$ is excluded in the cases of the TBM, GRA, GRB and HG neutrino mixing symmetry forms, respectively, at approximately $5 \sigma$, $4 \sigma, 4 \sigma$ and $3 \sigma$ C.L. with respect to the C.L. of the corresponding best fit values which all lie in the interval $J_{\mathrm{CP}}=(-0.034)-(-0.031)$. The best fit value for the BM (LC) form is much smaller and close to zero: $J_{\mathrm{CP}}=\left(-5 \times 10^{-3}\right)$. For the TBM, GRA, GRB and HG forms at $3 \sigma$ we have $0.020 \leq\left|J_{\mathrm{CP}}\right| \leq 0.039$. Thus, for these four forms the CP violating effects in neutrino oscillations are predicted to be relatively large and observable in the T2HK and DUNE experiments $[4,5]$, and

\footnotetext{
${ }^{10}$ For, e.g., $\sin ^{2} \theta_{12}=0.34$ allowed at $2 \sigma$ by the current data, we have $\cos \delta=-0.943$. Similarly, for $\sin ^{2} \theta_{12}=0.32$, $\sin ^{2} \theta_{23}=0.41$ and $\sin \theta_{13}=0.158$ we have [23]: $\cos \delta=-0.978$.

${ }^{11}$ Detailed results on the dependence of the predictions for $\cos \delta$ on $\sin ^{2} \theta_{12}, \sin ^{2} \theta_{23}$ and $\sin ^{2} \theta_{13}$ when the latter are varied in their respective $3 \sigma$ experimentally allowed ranges can be found in [33].
} 

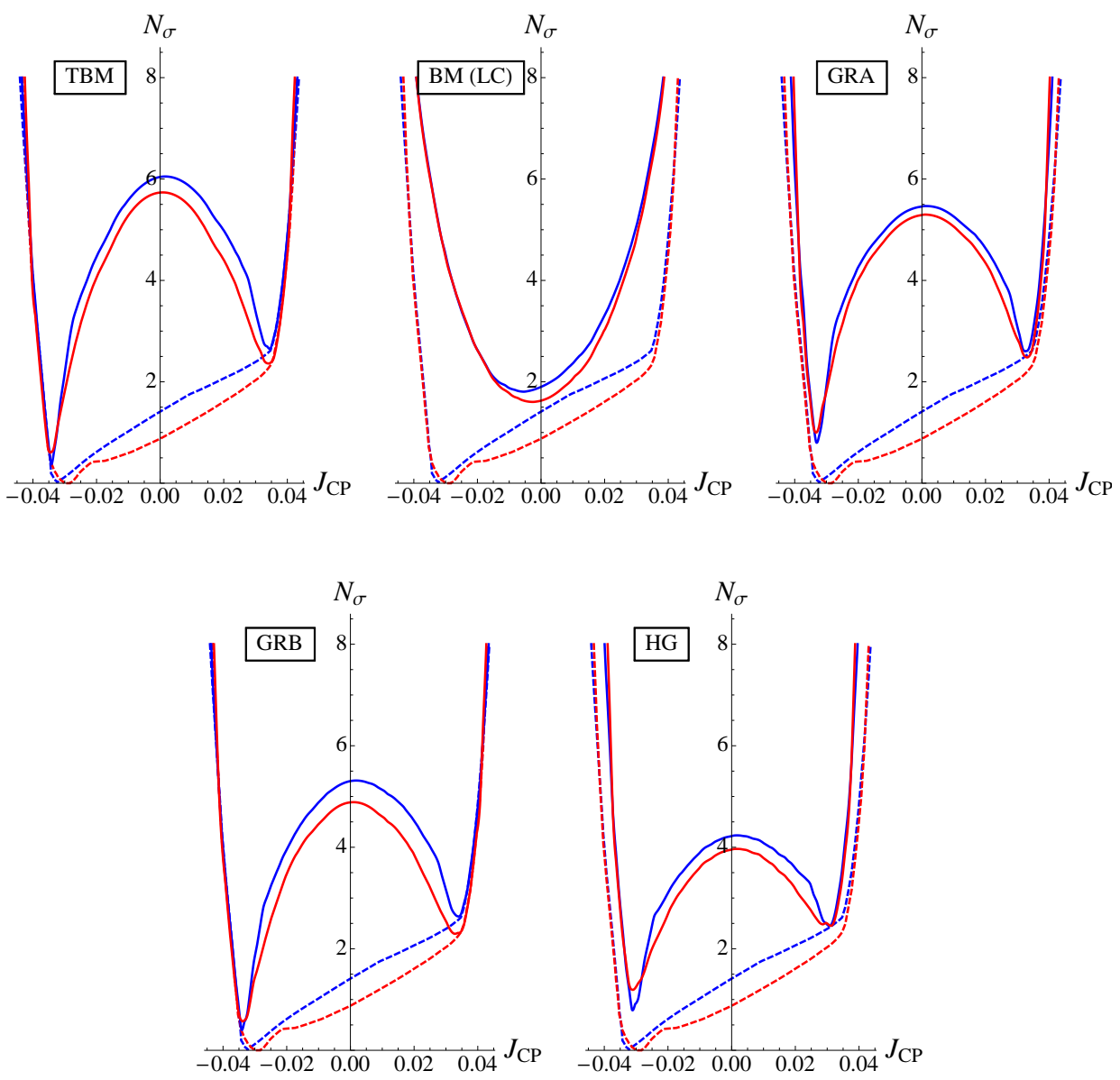

Figure 1: $N_{\sigma} \equiv \sqrt{\chi^{2}}$ as a function of $J_{\mathrm{CP}}$. The dashed lines represent the results of the global fit [12], while the solid lines represent the results we obtain for the TBM, BM (LC), GRA (upper left, central, right panels), GRB and HG (lower left and right panels) neutrino mixing symmetry forms. The blue (red) lines are for NO (IO) neutrino mass spectrum. (From ref. [33].)

perhaps even in T2K experiment [46]. These conclusions hold if one used in the analysis the results on the neutrino mixing parameters and $\delta$, obtained in the most recent global analysis [2].

In Fig. 2 (left panel) we present the results of the statistical analysis of the predictions for $\cos \delta$, namely the likelihood function versus $\cos \delta$ within the Gaussian approximation (see [33] for details) performed using the current b.f.v. of the mixing angles for NO neutrino mass spectrum given in ref. [12] and the prospective rather small $1 \sigma$ uncertainties i) of $0.7 \%$ on $\sin ^{2} \theta_{12}$, planned to be reached in JUNO experiment [47], ii) of $3 \%$ on $\sin ^{2} \theta_{13}$, foreseen to be obtained in the Daya Bay experiment [48], and iii) of $5 \%$ on $\sin ^{2} \theta_{23}$, expected to be reached in the currently running and future planned long baseline neutrino oscillation experiments. In the proposed upgrading of the currently taking data T2K experiment [46], for example, $\theta_{23}$ is estimated to be determined with a $1 \sigma$ error of $1.7^{\circ}, 0.5^{\circ}$ and $0.7^{\circ}$ if the best fit value of $\sin ^{2} \theta_{23}=0.50,0.43$ and 0.60 , respectively. This implies that for these three values of $\sin ^{2} \theta_{23}$ the absolute (relative) $1 \sigma$ error would be 0.0297 (5.94\%), $0.0086(2 \%)$ and $0.0120(2 \%)$. This error on $\sin ^{2} \theta_{23}$ is expected to be further reduced in the future planned T2HK [5] and DUNE [4] experiments. 

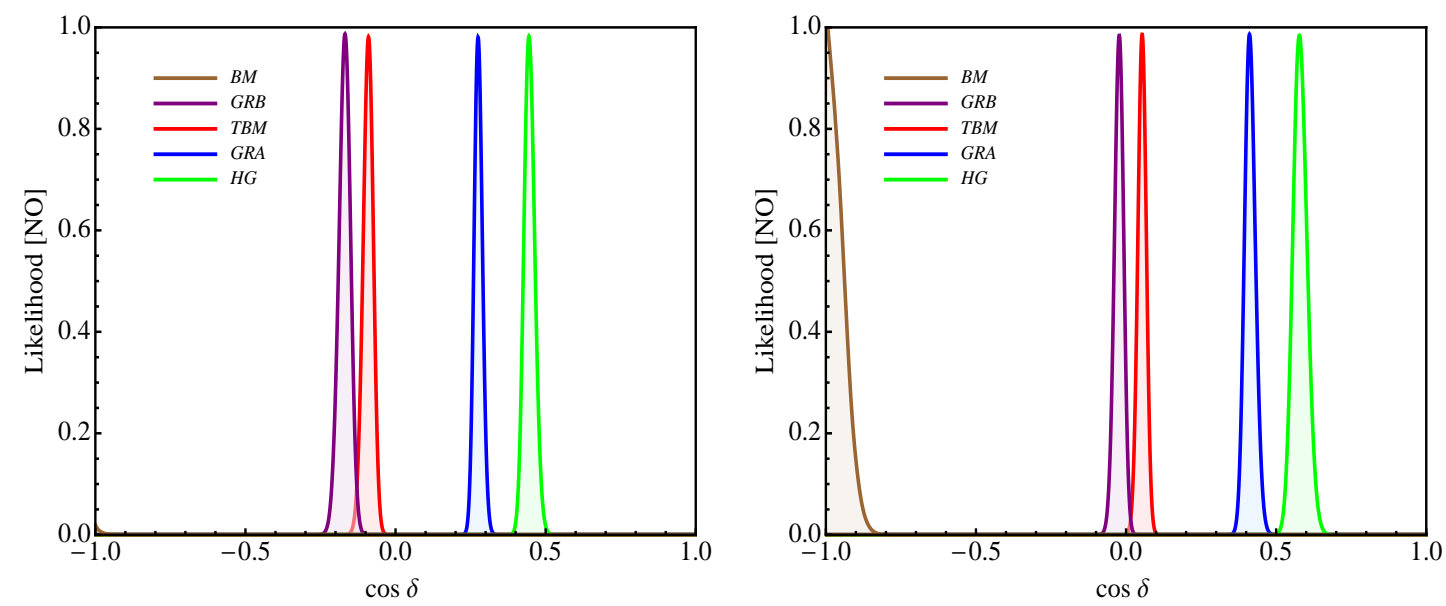

Figure 2: The likelihood function versus $\cos \delta$ for NO neutrino mass spectrum after marginalising over $\sin ^{2} \theta_{13}$ and $\sin ^{2} \theta_{23}$, for the TBM, BM (LC), GRA, GRB and HG symmetry forms of the mixing matrix $U_{v}^{\circ}$. The figure is obtained by using the prospective $1 \sigma$ uncertainties in the determination of $\sin ^{2} \theta_{12}, \sin ^{2} \theta_{13}$ and $\sin ^{2} \theta_{23}$ within the Gaussian approximation. In the left (right) panel $\sin ^{2} \theta_{12}$ is set to its b.f.v. of [12] 0.308 (is set to 0.332), the NO best fit values of the other angles are taken from [12]. (From ref. [33].)

As we have already remarked, the BM (LC) case is very sensitive to the b.f.v. of $\sin ^{2} \theta_{12}$ and $\sin ^{2} \theta_{23}$ and is disfavored at more than $2 \sigma$ for the b.f.v. found in [12] for the NO spectrum. This case might turn out to be compatible with the data for larger (smaller) measured values of $\sin ^{2} \theta_{12}$ $\left(\sin ^{2} \theta_{23}\right)$. This is illustrated in Fig. 2 (right panel).

The measurement of $\sin ^{2} \theta_{12}, \sin ^{2} \theta_{13}$ and $\sin ^{2} \theta_{23}$ with the quoted precision will open up the possibility to distinguish between the BM (LC), TBM/GRB, GRA and HG forms of $U_{v}^{\circ}$. Distinguishing between the TBM and GRB forms seems to require unrealistically high precision measurement of $\cos \delta{ }^{12}$. Assuming that $|\cos \delta|<0.93$, which means for $76 \%$ of values of $\delta$, the error on $\delta, \Delta \delta$, for an error on $\cos \delta, \Delta(\cos \delta)=0.10(0.08)$, does not exceed $\Delta \delta \lesssim \Delta(\cos \delta) / \sqrt{1-0.93^{2}}=16^{\circ}\left(12^{\circ}\right)$. This accuracy is planned to be reached in the future neutrino experiments like T2HK (ESS $v \mathrm{SB})[5,49]$. Therefore a measurement of $\cos \delta$ in the quoted range will allow one to distinguish between the TBM/GRB, BM (LC) and GRA/HG forms at approximately $3 \sigma$ C.L. if the precision achieved on $\sin ^{2} \theta_{12}, \sin ^{2} \theta_{13}$ and $\sin ^{2} \theta_{23}$ is the same as in Figs. 2. We are performing a more detailed study of the possibility to distinguish between BM (LC), TBM/GRB, GRA and HG forms of $U_{v}^{\circ}$ using the prospective data from DUNE and T2HK experiments [50].

In [24] we extended the analyses performed in [23, 33] by obtaining sum rules for $\cos \delta$ for $U_{\mathrm{PMNS}}$ having the general form given in eq. (2.3) and the following forms of $\tilde{U}_{e}$ and $U_{v}^{\circ}{ }^{13}$ :

C. $U_{v}^{\circ}=R_{23}\left(\theta_{23}^{v}\right) R_{12}\left(\theta_{12}^{v}\right)$ with $\theta_{23}^{v}=-\pi / 4$ and $\theta_{12}^{v}$ as dictated by TBM, BM, GRA, GRB or HG mixing, and i) $\tilde{U}_{e}=R_{13}^{-1}\left(\theta_{13}^{e}\right)\left(\Psi=\operatorname{diag}\left(1,1, e^{-i \omega}\right)\right)$, ii) $\tilde{U}_{e}=R_{23}^{-1}\left(\theta_{23}^{e}\right) R_{13}^{-1}\left(\theta_{13}^{e}\right)(\Psi=$

\footnotetext{
${ }^{12}$ Self-consistent models or theories of (lepton) flavour which lead to the GRB form of $U_{v}^{\circ}$ might still be possible to distinguish from those leading to the TBM form using the specific predictions of the two types of models for the neutrino mixing angles. The same observation applies to models which lead to the GRA and HG forms of $U_{v}^{\circ}$.

${ }^{13}$ We performed in [24] a systematic analysis of the forms of $\tilde{U}_{e}$ and $U_{v}^{\circ}$, for which sum rules for $\cos \delta$ of the type of eq. (2.6) could be derived, but did not exist in the literature.
} 
$\left.\operatorname{diag}\left(1, e^{-i \psi}, e^{-i \omega}\right)\right)$, and iii) $\tilde{U}_{e}=R_{13}^{-1}\left(\theta_{13}^{e}\right) R_{12}^{-1}\left(\theta_{12}^{e}\right)\left(\Psi=\operatorname{diag}\left(1, e^{-i \psi}, e^{-i \omega}\right)\right) ;$

D. $U_{v}^{\circ}=R_{23}\left(\theta_{23}^{v}\right) R_{13}\left(\theta_{13}^{v}\right) R_{12}\left(\theta_{12}^{v}\right)$ with $\theta_{23}^{v}, \theta_{13}^{v}$ and $\theta_{12}^{v}$ fixed by arguments associated with symmetries, and iv) $\tilde{U}_{e}=R_{12}^{-1}\left(\theta_{12}^{e}\right)\left(\Psi=\operatorname{diag}\left(1, e^{-i \psi}, 1\right)\right)$, and v) $\tilde{U}_{e}=R_{13}^{-1}\left(\theta_{13}^{e}\right)(\Psi=$ $\left.\operatorname{diag}\left(1,1, e^{-i \omega}\right)\right)$.

The sum rules for $\cos \delta$ were derived first for $\theta_{23}^{v}=-\pi / 4$ for the cases listed in point $\mathrm{C}$, and for the specific values of (some of) the angles in $U_{v}^{\circ}$, characterising the cases listed in point $\mathrm{D}$, as well as for arbitrary fixed values of all angles contained in $U_{v}^{\circ}$. In certain models with $\sin ^{2} \theta_{13}^{v} \neq 0, \sin ^{2} \theta_{23}$ is predicted to have specific values which differ significantly from those in case $\mathbf{B}$ [24]: $\sin ^{2} \theta_{23}=0.455$; or 0.463 ; or 0.537 ; or 0.545 , the uncertainties in these predictions being insignificant. Predictions for correlations between neutrino mixing angle values and/or sum rules for $\cos \delta$, which can be tested experimentally, were further derived in [25] for a large number of models based on $G_{f}=S_{4}, A_{4}, T^{\prime}$ and $A_{5}$ and: i) $G_{e}=Z_{2}$ and $G_{v}=Z_{n}, n>2$ or $Z_{n} \times Z_{m}, n, m \geq 2$;

ii) $G_{e}=Z_{n}, n>2$ or $G_{e}=Z_{n} \times Z_{m}, n, m \geq 2$ and $G_{v}=Z_{2}$;

iii) $G_{e}=Z_{2}$ and $G_{v}=Z_{2}$;

iv) $G_{e}$ is fully broken and $G_{v}=Z_{n}, n>2$ or $Z_{n} \times Z_{m}, n, m \geq 2$;

v) $G_{e}=Z_{n}, n>2$ or $Z_{n} \times Z_{m}, n, m \geq 2$ and $G_{v}$ is fully broken.

\section{Outlook}

The results obtained in refs. [23, 33, 24, 25, 30, 32, 11] and, e.g., in [29, 31, 34, 43] and in many other studies (quoted in the cited articles) show that a suffciently precise measurement of the Dirac phase $\delta$ of the PMNS neutrino mixing matrix in the current and future neutrino oscillation experiments, combined with planned improvements of the precision on the neutrino mixing angles, can provide unique information about the possible discrete symmetry origin of the observed pattern of neutrino mixing and, correspondingly, about the existence of new fundamental symmetry in the lepton sector. Thus, these experiments will not simply provide a high precision data on the neutrino mixing and Dirac CPV parameters, but will probe at fundamental level the origin of the observed form of neutrino mixing. These future data will show, in particular, whether Nature followed the the discrete symmetry approach for fixing the values of the three neutrino mixing angles and of the Dirac (and Majorana) CP violation phases of the PMNS neutrino mixing matrix. We are looking forward to these data and to the future exciting developments in neutrino physics.

Acknowledgements. I would like to thank M. Mezzetto and his colleagues for organising such a scientifically enjoyable Workshop. This work was supported in part by the INFN program on Theoretical Astroparticle Physics (TASP) and by the World Premier International Research Center Initiative (WPI Initiative, MEXT), Japan.

\section{References}

[1] K. Nakamura and S. T. Petcov, in C. Patrignani et al. [Particle Data Group], Chin. Phys. C 40 (2016) 100001.

[2] F. Capozzi et al., Phys. Rev. D (2017) [arXiv:1703.04471 [hep-ph]].

[3] I. Esteban et al., JHEP 1701 (2017) 087 [arXiv:1611.01514.

[4] R. Acciarri et al. [DUNE Collab.], arXiv:1512.06148, arXiv:1601.05471, arXiv:1601.02984.

[5] K. Abe et al. [Hyper-Kamiokande Proto-Collab.], PTEP 2015 (2015) 053C02 [arXiv:1502.05199]. 
[6] B. Pontecorvo, Zh. Eksp. Teor. Fiz. 33 (1957) 549 and 34 (1958) 247.

[7] Z. Maki, M. Nakagawa and S. Sakata, Prog. Theor. Phys. 28 (1962) 870.

[8] B. Pontecorvo, Zh. Eksp. Teor. Fiz. 53 (1967) 1717.

[9] S. M. Bilenky, J. Hosek and S. T. Petcov, Phys. Lett. B 94 (1980) 495.

[10] S. M. Bilenky and S. T. Petcov, Rev. Mod. Phys. 59 (1987) 671.

[11] J. T. Penedo, S. T. Petcov and A. V. Titov, arXiv:1705.00309.

[12] F. Capozzi et al., Phys. Rev. D 89 (2014) 093018.

[13] N. Cabibbo, Phys. Lett. B 72 (1978) 333.

[14] P.I. Krastev and S.T. Petcov, Phys. Lett. B 205 (1988) 84.

[15] C. Jarlskog, Z. Phys. C29 (1985) 491.

[16] P. Langacker et al., Nucl. Phys. B 282 (1987) 589.

[17] S.T. Petcov, Adv. High Energy Phys. 2013 (2013) 852987 [arXiv:1303.5819]; S.M. Bilenky, S. Pascoli and S.T. Petcov, Phys. Rev. D 64 (2001) 053010.

[18] S. Pascoli, S. T. Petcov and A. Riotto, Nucl. Phys. B 774 (2007) 1; Phys. Rev. D 75 (2007) 083511.

[19] P. Chen, G.-J. Ding and S.F. King, JHEP 1603 (2016) 206; C. Hagedorn and E. Molinaro, Nucl. Phys. B 919 (2017) 404.

[20] G. Altarelli and F. Feruglio, Rev. Mod. Phys. 82 (2010) 2701.

[21] H. Ishimori et al., Prog. Theor. Phys. Suppl. 183 (2010) 1 [arXiv:1003.3552].

[22] M. Tanimoto, AIP Conf. Proc. 1666 (2015) 120002.

[23] S. T. Petcov, Nucl. Phys. B 892 (2015) 400 [arXiv:1405.6006].

[24] I. Girardi, S. T. Petcov and A. V. Titov, Eur. Phys. J. C 75 (2015) 345.

[25] I. Girardi et al., Nucl. Phys. B 902 (2016) 1.

[26] D. Marzocca et al., JHEP 1305 (2013) 073.

[27] A. de Gouvea and H. Murayama, Phys. Lett. B 747 (2015) 479 and B 573 (2003) 94; L. Hall, H. Murayama and N. Weiner, Phys. Rev. Lett. 84 (2000) 2572.

[28] G. C. Branco, L. Lavoura and M. N. Rebelo, Phys. Lett. B 180 (1986) 264.

[29] F. Feruglio, C. Hagedorn and R. Ziegler, JHEP 1307 (2013) 027; M. Holthausen, M. Lindner and M. A. Schmidt, JHEP 1304 (2013) 122.

[30] I. Girardi et al., JHEP 1402 (2014) 050. J. N. Lu and G. J. Ding, Phys. Rev. D 95 (2017) 015012.

[31] P. Ballett, S. Pascoli and J. Turner, Phys. Rev. D 92 (2015) 093008. J. Turner, Phys. Rev. D 92 (2015) 116007.

[32] I. Girardi, S. T. Petcov and A. V. Titov, Nucl. Phys. B 911 (2016) 754.

[33] I. Girardi, S. T. Petcov and A. V. Titov, Nucl. Phys. B 894 (2015) 733; Int. J. Mod. Phys. A 30 (2015) 1530035 [arXiv:1504.02402].

[34] A. D. Hanlon, S. F. Ge and W. W. Repko, Phys. Lett. B 729 (2014) 185.

[35] P. Ballett et al., Phys. Rev. D 89 (2014) 016016. 
[36] A. Meroni, S. T. Petcov and M. Spinrath, Phys. Rev. D 86 (2012) 113003; S. Antusch et al., Nucl. Phys. B 877 (2013) 772; C. Hagedorn and D. Meloni, Nucl. Phys. B 862 (2012) 691.

[37] P. F. Harrison, D. H. Perkins and W. G. Scott, Phys. Lett. B530 (2002) 167; Z. z. Xing, Phys. Lett. B533 (2002) 85; see also: L. Wolfenstein, Phys. Rev. D 18 (1978) 958.

[38] S. T. Petcov, Phys. Lett. B 110 (1982) 245.

[39] F. Vissani, hep-ph/9708483; V. D. Barger et al., Phys. Lett. B437 (1998) 107.

[40] A. Datta, F. S. Ling and P. Ramond, Nucl. Phys. B671 (2003) 383; L. L. Everett and A. J. Stuart, Phys. Rev. D79 (2009) 085005; Y. Kajiyama, M. Raidal and A. Strumia, Phys. Rev. D76 (2007) 117301.

[41] W. Rodejohann, Phys. Lett. B 671 (2009) 267; A. Adulpravitchai, A. Blum and W. Rodejohann, New J. Phys. 11 (2009) 063026.

[42] C. H. Albright, A. Dueck and W. Rodejohann, Eur. Phys. J. C 70 (2010) 1099; J. E. Kim and M. S. Seo, JHEP 1102 (2011) 097.

[43] G. Altarelli, F. Feruglio and L. Merlo, JHEP 0905 (2009) 020.

[44] P. H. Frampton, S. T. Petcov and W. Rodejohann, Nucl. Phys. B 687 (2004) 31.

[45] J. Gehrlein et al., Nucl. Phys. B 890 (2014) 539.

[46] K. Abe et al., arXiv:1609.04111 [hep-ex].

[47] F. An et al. [JUNO Collab.], J. Phys. G 43 (2016) no.3, 030401 [arXiv:1507.05613];

[48] Daya Bay Collaboration, arXiv:1309.7961.

[49] E. Baussan et al. [ESSnuSB Collab.], Nucl. Phys. B 885 (2014) 127.

[50] S.K. Agarwalla et al., in preparation. 